\title{
An Evidence-Based Review of Treatment-Related Determinants of Patients' Nonadherence to HIV Medications
}

\author{
Mark J. Atkinson, M.Ed., Ph.D.,2 and Jeffrey J. Petrozzino, M.D., Ph.D. ${ }^{3}$
}

\begin{abstract}
Patients' adherence to antiretroviral medications is a primary determinant of both the effectiveness of treatment and the clinical course of HIV/AIDS. This empirical review is intended to compare the relative importance of patient and treatment characteristics on nonadherence behavior and the impact of nonadherence on treatment failure. Articles cited in PubMed and published between 2006 and June $2008(n=200)$ were reviewed to select those that address patient or treatment characteristics associated with nonadherence. Twenty-two articles were selected that provided odds ratio or hazard ratio statistics that quantified predictors of patients' level of nonadherence (e.g., $<80 \%, 80 \%-95 \%$ and $>95 \%$ ). Results were summarized using random effects meta-analytic models. Predictors of nonadherence were divided into four predictive clusters (clinical predictors, comorbid predictors, treatment competence predictors, and dosing predictors). The summary odds ratios (ORs) of nonadherence for each cluster (in order of strength) were treatment competence 2.0 (95\% confidence interval [CI]: 1.6-2.6), clinical predictors 1.6 (95\% CI: 1.4-1.8), comorbid predictors 1.6 (95\% CI: $1.4-1.8)$, and dosing predictors 1.5 (95\% CI: 1.3-1.7). The effect of nonadherence on treatment failure supported the findings of two prior empirical reviews (OR 2.0, 95\% CI: 1.6-2.5). Within dosing predictors, a pill burden of more versus less than 10 pills per day was associated with a much higher odds of nonadherence than twice versus once daily dosing or small differences in the number of types of antiretroviral treatments in a regimen. These results provide insight into the relative importance of various determinants of patient nonadherence that may inform the design of patient educational initiatives and initiatives to simplify treatment regimens.
\end{abstract}

\section{Introduction}

A BRIEF REPORT on improvements in virologic response to antiretroviral therapy (ART) occurring between 1996 and 2002 suggests that improved clinical outcomes are the result of two interrelated factors: improved antiviral treatments and a greater focus on factors affecting patients' medication adherence. ${ }^{1}$ Particularly important advances in the medications used to treat HIV/AIDS occurred after 1999 with the development of protease inhibitor (PI)-boosted and nonnucleoside reverse transcriptase inhibitor (NNRTI) regimens, both of which relaxed the requirement that more than $95 \%$ adherence be observed to prevent viral breakthrough observed in patients following strictly PI-based regimens. On the side of clinical intervention and drug development, a greater focus on medication adherence, improved tolerability of ART, and ease of dosing has resulted in more tolerable formulations and raised patients' awareness of the importance of medica- tion adherence. As a result of the central role that ART adherence plays in preventing serious long-term clinical consequences of HIV/AIDS, the field of HIV treatment is a forerunner in the emerging science of treatment adherence.

There have been two major reviews of medication nonadherence and HIV clinical outcomes over the last 4 years. One performed by Simoni et al. $^{2}$ reports on 77 articles published between 1996 and 2004. A second meta-analysis by Nieuwkerk and Oort ${ }^{3}$ reports on 65 articles published between 1996 and 2003. Both articles suggest that the risk of HIV regimen failure is doubled among patients who are nonadherent to treatment. The meta-analysis by Nieuwkerk and Oort examined the adherence predictors of plasma HIV RNA concentration outcomes among adult patients prescribed ART using self-reported adherence measures. Across all studies, the pooled OR of detectable plasma viral load in nonadherent patients was 2.3 (95\% CI: 2.0-2.7) compared to adherent patients.

\footnotetext{
${ }^{1}$ PRO-Spectus LLC, San Diego, California.

${ }^{2}$ Health Services Research Center, University of California at San Diego, San Diego, California.

${ }^{3}$ Compara Biomedical, Orlando, Florida.
} 
Simoni and colleagues ${ }^{2}$ also report an overall doubling of the risk for viral breakthrough among nonadherent patient samples, although they suggest that a number of complexities in the field hinder clear specification of a precise relationship between nonadherence and proximal clinical outcomes. These include: (1) the absence of standard approaches to define and operationalize adherence; (2) great variability in the time periods over which adherence data are observed (days to years); (3) the wide variety of methods used to assess medication adherence; and (4) the great diversity of study samples. In addition to specific measurement and methodological problems, biologically based interactions between medication adherence, viral replicative capacity, and medication pharmacokinetics in vivo preclude clear specification of adequate versus poor adherence behavior. Since publication of these reviews, some progress has been made to address these concerns.

\section{Control of HIV replication and viral resistance}

Maintaining a therapeutic plasma level of multiple antiretroviral agents is the primary determinant of effective HIV suppression and prevention of viral breakthrough. $\mathrm{C}_{\text {trough }}$ levels describe the lowest plasma concentration between dosing cycles, and are affected by inadequate adherence to HIV medication regimens as well as drug metabolism. Because poor adherence to HIV medication often leads to incomplete suppression of viral replication, plasma viral load (described by log of HIV-1 RNA or viral copies per microliter) is the most easily observed and immediate response to treatment failure. As viral breakthrough intensifies, a patient's CD4 ${ }^{+}$lymphocyte count drops. This decline has a more direct relationship to clinical symptoms and AIDS-related morbidities than viral load. ${ }^{4}$ For this reason, the clinically based Centers for Disease Control and Prevention (CDC) criteria for HIV disease progression is based primarily on $\mathrm{CD}^{+}$count. $^{5}$

These antiretroviral agents belong to one of five main HIV treatment classes: Binding or entry inhibitors (CCR5), PIs; nucleoside analogue reverse transcriptase inhibitors (NARTIs or NTRTIs), NNRTIs, and the integrase inhibitors (IIs). The class membership is defined by the treatment mechanism of action and by which step in the HIV virus adsorption, integration, or replication pathway is interrupted by treatment. ${ }^{5}$

In the 1990s combinations of HIV therapeutic agents, appropriately named highly active antiretroviral therapy (HAART), were shown to be more effective at preventing viral replication and HIV/AIDS-related morbidity than treatment regimens containing a single medication class. The use of multiple classes creates a series of simultaneous barriers to viral replication at multiple points in the replication pathway. In order for the HIV virus to overcome and "breakthrough" multiple treatment barriers, a greater number of resistance mutations would have to occur in close temporal proximity.

Resistant viral strains can be either acquired at the time of infection or spontaneously occur through a process of selection in the presence of only partially effective blockades to viral synthesis. Medication levels below minimally therapeutic plasma concentrations $\left(C_{\min }\right)$ allow for slow viral replication of resistant strains. The capacity of HIV to replicate in the presence of suboptimal levels of medication (i.e., rep- licative capacity) results in the emergence of viral resistance. ${ }^{6}$ Viral resistance has serious treatment implications. With few exceptions, previous failure of a particular type of HIV medication renders all others in its class ineffective as a future treatment options. ${ }^{7}$ Moreover, such resistance can be passed onto other individuals, with serious implications for available treatment options.

Until very recently, the need for multiple medication classes to maintain an effective blockade, along with different dosing schedules due to varying $\mathrm{pK}$ characteristics have meant that patients are typically required to take a large number of different types of pills, several times a day. Further complicating the situation is the need to follow strict dietary requirements, since therapeutic dose levels are affected by gastrointestinal absorption rates. As a result, patients are asked to adhere to strict administration requirements in terms of timing, dosing, and dietary conditions, all of which over time, are associated with various types of nonadherence events. ${ }^{8}$

\section{Challenges facing definition of optimal HIV treatment nonadherence}

Strict adherence to a medication regimen is necessary in order for medications to be clinically effective and result in a sustained suppression of HIV viral replication. ${ }^{9-11}$ Therefore, drugs that are associated with better adherence are clinically favored, and factors affecting adherence are important when deciding on an appropriate anti-HIV treatment regimen. ${ }^{12}$ As an example, PI-sparing medication regimens are clinically chosen over those that contain a PI, because PIbased regimens are typically associated with more severe side effect profiles, dietary administration requirements, and lower levels of medication adherence. ${ }^{13}$

The level of treatment nonadherence affects not only whether viral breakthrough and a decline in $\mathrm{CD} 4^{+}$count occurs within a given time period, it also affects the length of time that ART continues to work after initial viral suppression. ${ }^{14,15}$ Moreover, a number of studies have observed a doseresponse phenomenon between nonadherence and decrements in the durability of treatment response assessed by viral load and $\mathrm{CD}^{+}{ }^{+}$count. $^{16}$ For example, the time to virologic failure has been shown to be associated with the frequency of dosing in a lopinavir/ritonavir PI-based regimen, likely because of worse diarrhea in twice-daily than once-daily dosing schedules. ${ }^{15}$ While a primary goal of "medication simplification" is reduced dosing requirements, this not always easily achieved. $^{17}$

Based on the pharmacokinetics of medications and the associated plasma clearance rates, some medication regimens maintain an effective barrier to viral replication (plasma $C_{\min }$ levels) even when patients are less temporally consistent with medication use. For example, efavirenz has a therapeutic dose half-life of 40 hours, allowing for longer effective viral suppression than other antiretrovirals. ${ }^{18}$ As a result, researchers will occasionally use different nonadherence criteria based on the pharmacologic characteristics of a particular medication regimen and choose nonadherence criteria at the point where nonadherence becomes problematic.

Also frustrating attempts to settle on a standard adherence threshold is the observation that the HIV virus begins to replicate at different drug plasma concentrations based on the 
medication and drug mechanism. ${ }^{6}$ For example, boosted PIs may allow for HIV replicative capacity just below effective therapeutic dosing, thereby requiring more strict adherence, while HIV replicative capacity in patients on an NNRTI regimen may begin at much lower subtherapeutic plasma concentrations and be more forgiving of minor nonadherence.

\section{Determinants of medication nonadherence}

Nonadherence is the extent to which a person's use of medication fails to coincide with medical advice. There have been a number of recent empirical reviews of the predictors of medication nonadherence and the impact of various levels of nonadherence on viral breakthrough. These reviews identify certain patient characteristics are associated with medication nonadherence, such as drug addiction, psychiatric comorbities (e.g., anxiety, depression) and poor social support ${ }^{19-21}$; while clinical variables of high baseline viral load, low CD4 counts and coinfections are associated with poorer treatment outcomes. ${ }^{19}$ Moreover, levels of medication nonadherence and rates of treatment failure can be reduced by providing comprehensive and structured clinical management services, particularly those that address patients' problems with drug addiction and limited psychosocial resources. ${ }^{20}$ Such findings often result in the elimination of more problematic subpopulations such as drug users and homeless from trials and research summaries. ${ }^{22}$

When patients are asked to give various reasons for missing doses and taking less ART medication than prescribed, these reasons generally fall into four categories: conflicts with daily routines; poor medication tolerability; regimen complexity or burden; and financial and access barriers to treatment. ${ }^{23-25}$ More specifically, treatment characteristics that contribute to nonadherence are the frequency and severity of side effects, as well as interference of administration requirements with daily routines (e.g., dietary requirements, dosing frequency, and the number and of medications in the regimen). ${ }^{8,15,26}$ Drug development strategies designed to simplify regimens therefore seek to reduce the common reasons for nonadherence and ultimately improve clinical outcomes. ${ }^{13}$

Because of the complexity of treatment regimens, inexperienced patients may be less well informed and be inadvertently nonadherent or ineffective with HIV medication use. ${ }^{27,28}$ Adherence to complex medication regimens may also be compromised by mental health comorbidities associated with poor comprehension, forgetfulness, and functional disorganization. Finally, the therapeutic alliance between patients and their caregivers can affect adherence by influencing patients' expectations and knowledge about effective medication use. To date, however, there has not been a systematic comparative review of the effects of either medication characteristics or patients' level of administration competence, both of which appear to affect medication adherence.

\section{Measurement of medication adherence}

Accurate assessment of adherence behavior is an essential, yet enigmatic component of any attempt to study adherence to antiretroviral medications. ${ }^{2}$ There is no gold standard for adherence assessment. Various methods have been developed to measure antiretroviral adherence: lab assessments of biologic levels of drugs or drug metabolites; the results of pill counts, typically taken at the time of clinic visit; the electronic recording of when medication bottles are opened; patients' self-report of their level of adherence to medication schedules ${ }^{29}$; pharmacy refill records; and provider reports of directly observed treatment (DOT).$^{30}$ Each of these methods has its strengths and limitation in terms of the degree of random error and types of systematic biases that influence estimates of medication nonadherence. ${ }^{31-33}$

The precision of any nonadherence point estimate will be influenced by measurement error and systematic response bias associated with the assessment method. An example is the systematic bias observed using self-reported medication adherence measures, where the effects of response bias appears to result in greater self-reported nonadherence than clinical assessments, ${ }^{34}$ which may explain why the concordance between electronic drug monitoring and self-reports are higher among less adherent groups than more adherent individuals. ${ }^{35}$ Such biases likely account for low sensitivity estimates associated with many self-report measures, although the specificity (i.e., accurate identification of those that report nonadherent behavior against a viral load criterion) appears to be very good. ${ }^{36}$ To balance such drawbacks, which are inherent in any method, some researchers recommend using multiple approaches. These include a "Composite Adherence Score" based on a primary measure (e.g., the Medication Event Monitoring System [MEMS] cap data) and additional measures (e.g., pill counts or interview data) to supplement missing or defective MEMS cap data. ${ }^{37}$

Operational definitions and considerations. The most commonly reported ways that medication nonadherence statistics are: taking adherence (refill adherence); dose adherence (correct adherence to dose); schedule/timing adherence; dietary adherence; differential adherence (reported for each medication in a regimen); and time updated adherence (rolling average of adherence over time). Assessments that allow more fine-grained analysis permit computation of a number of different types of nonadherence estimates, and raise some interesting questions. For example, patients who are fully dose compliant may still be nonadherent with respect to dietary or scheduling requirements; which forms of nonadherence are most important to the research questions being asked? Also, which types of nonadherence statistics are most appropriate to use with the identified patient samples and treatments they are taking? As the number of compliance criteria increase, summary estimates of the pooled nonadherence in the sample should be considered carefully. ${ }^{38}$

Nonadherence cut-points are often defined and reported as ranges, typically more than $95 \%, 95 \%-80 \%$, and less than $80 \%$ adherence. In some studies these cut-points are chosen based on the degree of nonadherence that medication regimens can withstand before failing. In other studies, a standard scoring algorithm is available and applied for a particular assessment method. Still other researchers avoid the use of cut-point criteria altogether and report the effect sizes associated with incremental changes in nonadherence (e.g., $100 \% 99 \%-90 \%, 89 \%-80 \%$, etc.) on clinical outcomes. This particular approach is very informative but provides quite small effect size estimates in comparison to the cut-point method. Variation across studies in which cutpoint criteria are applied makes comparisons of adherence 


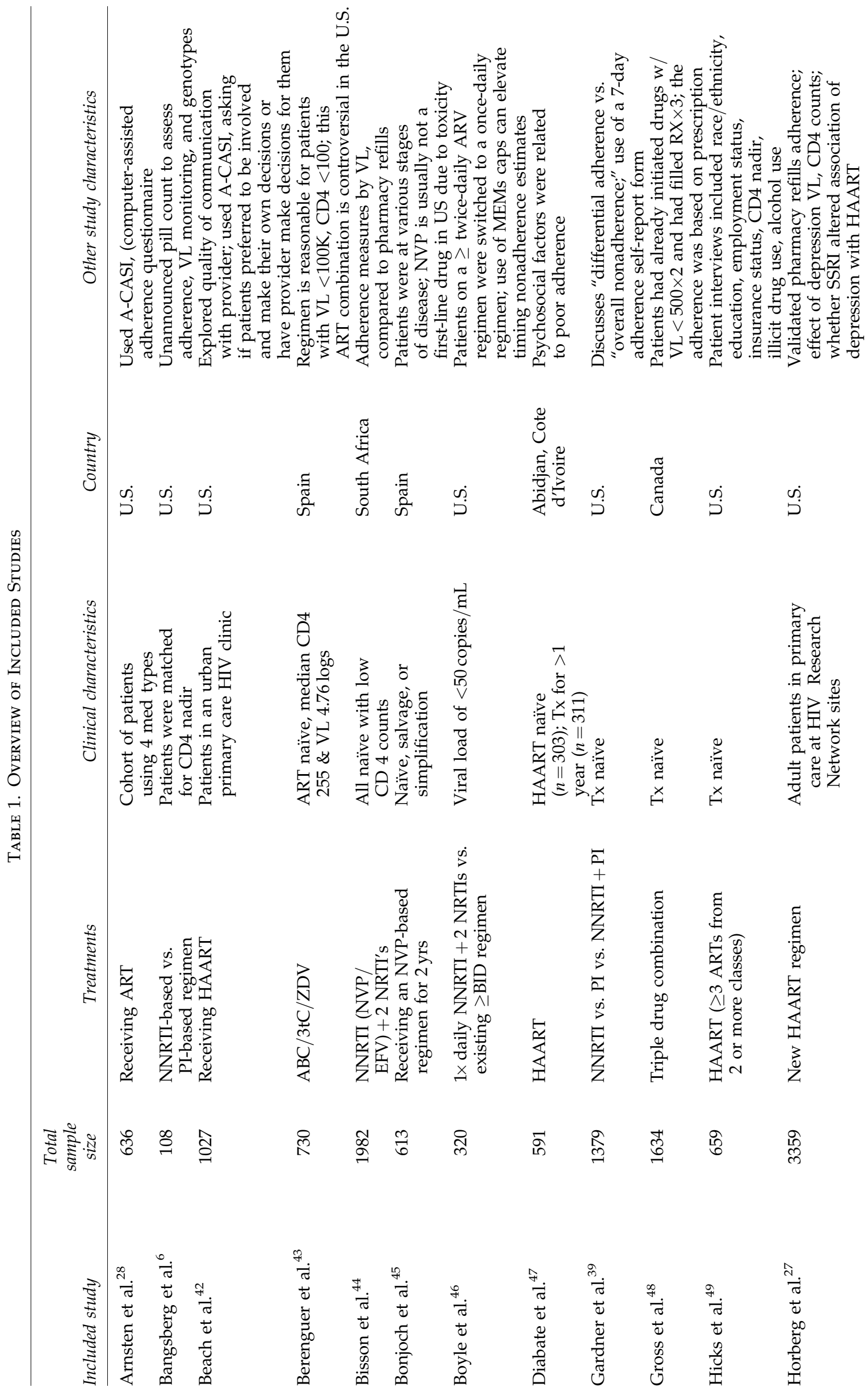




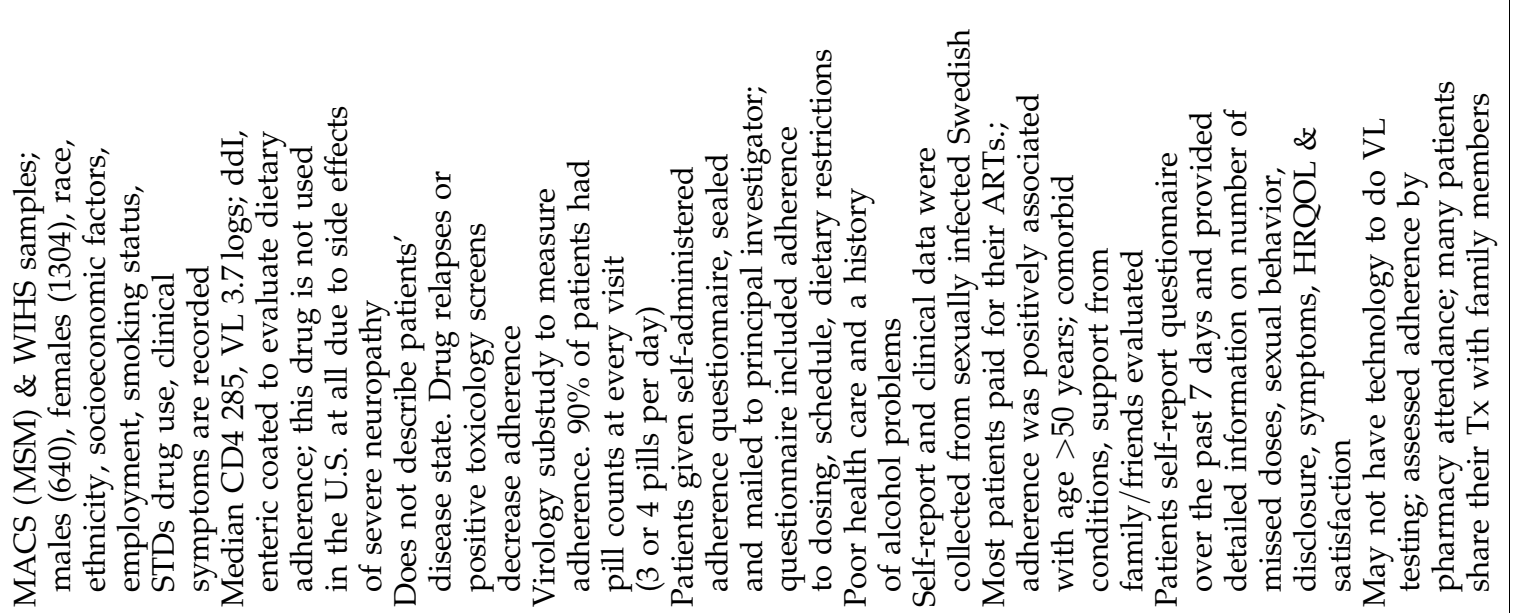

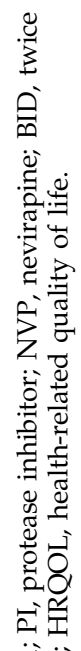

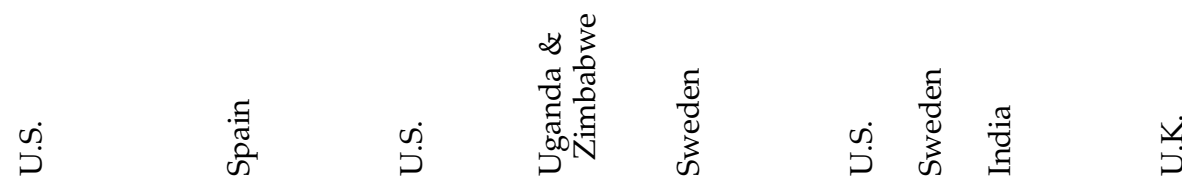
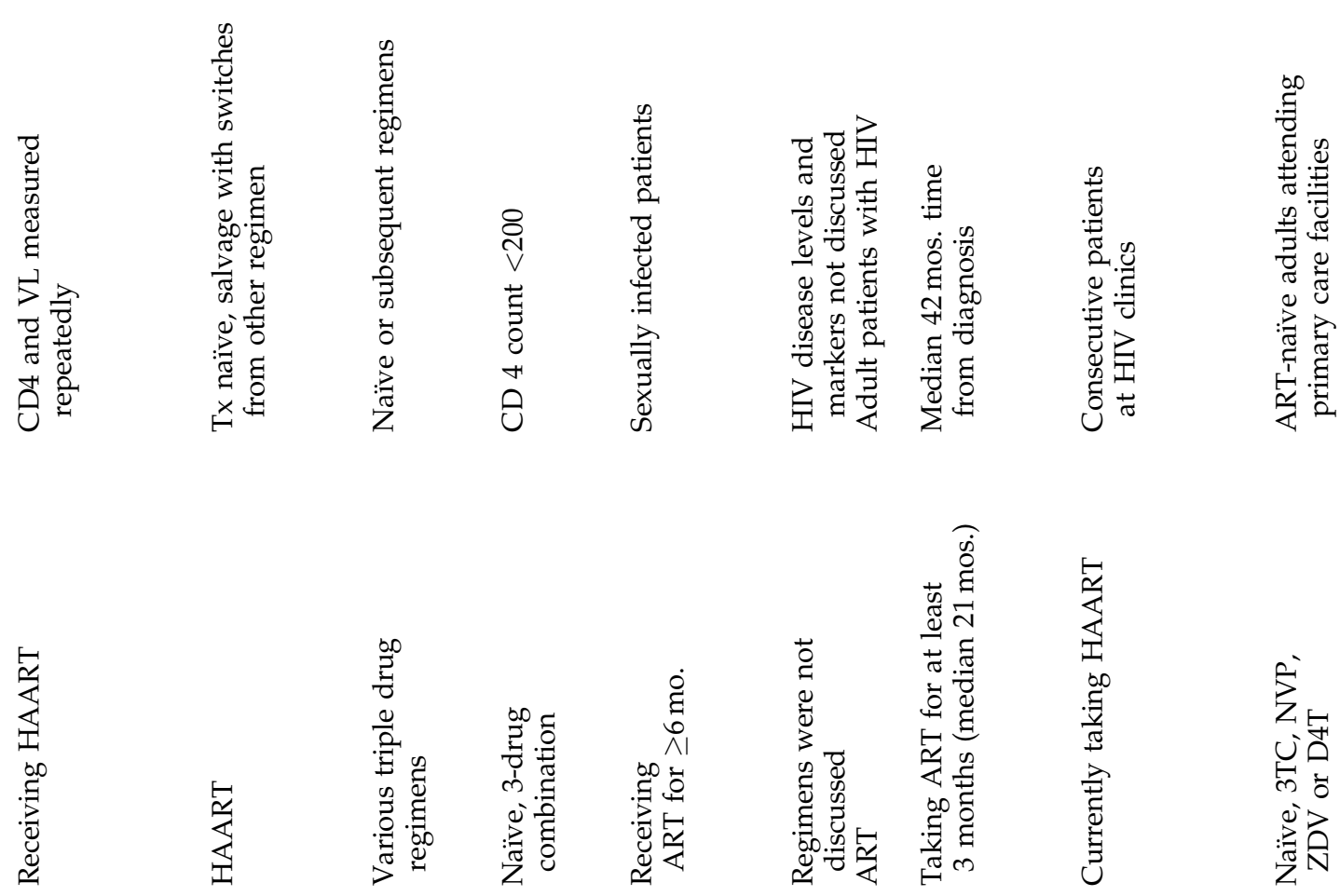

竞

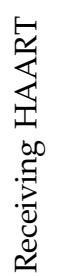

素

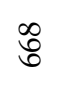

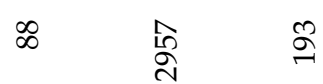

त

ธั

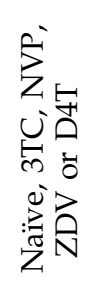

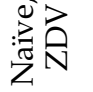

$\stackrel{4}{4}$

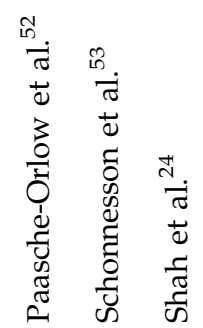

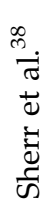

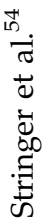

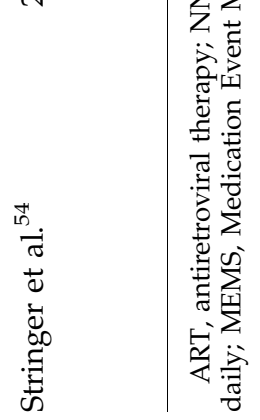


statistics difficult, and can attenuate a pooled effect size estimate across studies.

Adherence statistics can also be reported by regimen or by each individual medication within the regimen. Although this distinction is becoming less important as medications move towards combination dosage forms, "differential" nonadherence rates for each medication in a regimen can provide important information on drug performance in vivo. ${ }^{39}$

Timing of the observational windows. The length of time nonadherence is assessed varies greatly across studies; from self-reported or observed behaviors occurring over the course of several days, to less detailed nonadherence assessments using pharmacy script refills over the course of many months or years. Each comes with a unique set of adherence metrics and computational methods to delineate levels of nonadherence.

Longer cohort pharmacy script studies of patients in large HIV treatment registries use mixed populations that differ in terms of the length of time patients have been on ART and what types of treatments they have taken. Often such studies cannot feasibly resolve the large number of interactions between diverse treatment regimens and treatment histories. In contrast, prospective adherence studies that use repeated, short observational windows are typically more detailed and can account for both medication and patient characteristics when modeling the impact of nonadherence on clinical outcomes. As discussed, such detail allows for operational definitions of adherence to include such things as missed doses, lax dosing schedules, ${ }^{15}$ and failure to observe dietary restrictions. ${ }^{38,40,41}$

Longitudinal models of clinically important outcomes such as virologic suppression and virologic failure over time reveal the temporal effects of a dose-response relationship with level of nonadherence, ${ }^{14}$ such results are often displayed as a survival curve. The implication is that the points in time that assessments are made after patients' start therapy can influence strength of the observed relationships.

\section{Objectives of the Study}

This empirical review covers recent HIV treatment literature that has examined the relationship between the reasons for patients' nonadherence to HIV medication and treatment failure, and was intended to compare the relative influence of psychosocial and treatment factors on patient nonadherence. It was anticipated that a comparative evaluation of the various causes of nonadherence behaviors would help inform the educational and clinical programs aimed at promoting patient adherence to HIV treatment regimens. The key questions addressed in the study were:

- What patient characteristics and medication attributes affect HIV medication nonadherence?

- What types of clinical conditions and comorbidities affect nonadherence and short-term indicators of HIV treatment failure?

- What observations can be made with respect to the relative effect sizes between various predictors of nonadherence and medication failure?

\section{Methods}

A literature search of PubMed/MEDLINE was used to identify articles published between 2006 and 2008 that addressed various adherence predictive clusters. Articles were chosen that provided odds ratios (OR) and hazard ratios (HR) as effect size estimates of the relationships between treatment nonadherence and viral breakthrough or treatment-related predictors of nonadherence and nonadherence.

\section{Search and selection strategies}

PubMed/MEDLINE was searched for potential articles using the following three search strings:

Search string \#1: Measurement of nonadherence (resulted in 125 articles)

((("HIV"[Mesh]) OR (human immunodeficiency virus)) AND (adhere* OR complian*) AND (viral load OR breakthrough OR break through OR "Drug Resistance, Viral"[Mesh] OR "CD4 Lymphocyte Count"[Mesh])) AND ((composite adherence score) OR (ACTG adherence) OR (AIDS clinical trials group adherence) OR (pill count*) OR (prescription fill*) OR ("Drug Monitoring"[Mesh]) OR ("Self Assessment (Psychology)" [Mesh]) OR (mems caps) OR (adherence questionnaire) OR (self report* OR self-report* OR patient report* OR patientreport*) OR (drug level)) AND ("last 3 years"[PDat] AND (Humans[Mesh]) AND (English[lang]))

Search string \#2: Determinants of nonadherence (resulted in 51 articles)

((("HIV"[Mesh]) OR (human immunodeficiency virus)) AND (adhere* OR complian*) AND (side effects OR convenience OR effectiveness OR efficacy) AND (viral load OR breakthrough OR break through OR "Drug Resistance, Viral"[Mesh] OR "CD4 Lymphocyte Count"[Mesh])) AND ((composite adherence score) OR (ACTG adherence) OR (AIDS clinical trials group adherence) OR (pill count*) OR (prescription fill*) OR ("Drug Monitoring"[Mesh]) OR ("Self Assessment (Psychology)"[Mesh]) OR (mems caps) OR (adherence questionnaire) OR (self report* OR self-report* OR patient report* OR patientreport*) OR (drug level)) AND ("last 3 years"[PDat] AND (Humans[Mesh]) AND (English[lang]))

Search String \#3: Dosing effects on nonadherence (resulted in 36 articles)

(("HIV"[Mesh]) OR (human immunodeficiency virus)) AND ((adhere* OR complian*) OR (composite adherence score) OR (ACTG adherence) OR (AIDS clinical trials group adherence) OR (adherence questionnaire)) AND ((pill burden) OR (pill number) OR (number of pills)) AND ("last 3 years"[PDat] AND (Humans[Mesh]) AND (English[lang]))

\section{Article selection and data extraction}

Candidate articles $(n=200)$ were identified that were published in English between January, 2006 and June, 2008. Only abstracts that included, or suggested the presence of ORs or HRs related to psychosocial or treatment characteristics were selected for review. This selection process resulted in 91 full-text articles being reviewed further, with 23 of these identified as containing OR or HR data that could be included in the empirical review (Table 1).

Because the dimensions assessed across studies varied widely, unadjusted bivariate OR and HR values were sought whenever available, although it was observed that the adjusted statistics rarely differed much from the unadjusted 
Table 2. Classification Codes for Stratification of Analyses Predicting Nonadherence

\begin{tabular}{lll}
\hline & Predictive clusters & \multicolumn{1}{c}{ Nonadherence } \\
\hline Clinical Codes & Dosing Codes & Adherence Assessment Codes \\
1. Baseline VL & 1. \# of pills, pill burden & 1. Self-report measure or interview \\
2. Baseline resistance & 2. \# of medication classes & 2. Dispensing devices \\
3. AIDS-defining condition & 3. Regimen complexity, dietary, dosing & 3. Plasma, urine assays \\
4. Low CD4 ${ }^{+}$count & 4. Ease of administration, use & 4. Pill count \\
5. Length of time on Tx & Tx Competence Codes & 5. Pharmacy scripts, refills \\
6. Previous tx failure & 1. Tx experience, naïve & 6. Directly observed Tx \\
7. Adverse events, side effects & 2. Tx self-efficacy & 7. Physician reported \\
8. Comparison of Tx regimens & 3. Tx education (Tx options, adherence) & Adherence level codes \\
& 4. Tx knowledge & 1. Full or excellent $(100 \%)$ \\
Comorbidity codes & 5. Tx beliefs, attitudes & 2. Adequate $(>95 \%$ or $80 \%-95 \%)$ \\
1. Depression, anxiety & 6. Doctor, patient, clinic relationships & 3. Inadequate $(<80 \%)$ \\
3. Substance abuse, alcohol, drugs & & \\
4. Psychological conditions, symptoms & &
\end{tabular}

VL, viral load; $T x$, treatment.

values. In cases where unadjusted values were not provided or extractable, multivariate values were used.

\section{Statistical methods}

Some ORs were inverted by computation using the original dataset so that ORs were expressed consistently across studies. Close attention was paid to making sure that the correct attributions were made with respect to interpretation based on the reference group. For example Hicks et al. ${ }^{49}$ reported an OR of 0.45 for good versus inadequate adherence associated with persons who reported hazardous drinking compared to those who did not drink. This statistic was reversed so that hazardous drinking was associated with an OR for inadequate adherence.

A spreadsheet was created with each line conceptually classified based on the type of adherence assessment method used, the type of predictor and outcome groups used, and the predictive cluster the effect size estimate belonged within. Table 2 presents the codes that were evolved within each predictive cluster based on the data that were available within the identified studies.
A random effects model was used to estimate the summary $\mathrm{OR} / \mathrm{HR}$ and $95 \%$ confidence intervals associated with each predictive cluster.

\section{Results}

OR/HR data gleaned from the 22 articles were grouped according to one of the four predictive clusters of nonadherence (Tables 3-6) or nonadherence predictors of viral break-through (Table 2).

The clinical predictors of nonadherence presented in Table 3 included the number and types of adverse events, baseline viral load, baseline CD4+ count, and the use of a PI regimen (Table 3). An overall OR of 1.6 (95\% C.I.: 1.4-1.8) was reported for this cluster.

Comorbid predictors of nonadherence included problematic alcohol and drug abuse, depression, and psychological symptoms (Table 4). On average individuals with these comorbid conditions had 1.6 (95\% CI: 1.4-1.8) greater odds of treatment nonadherence than those without such concerns. Because multiple comorbidities are not uncommon, the

Table 3. Clinical Predictors of Medication Nonadherence ${ }^{a}$

\begin{tabular}{|c|c|c|}
\hline Study name & $\begin{array}{l}\text { Odds } \\
\text { ratio }\end{array}$ & Comparison \\
\hline $\begin{array}{l}\text { 1. Lucas et al., } 2007 \\
\text { 2. Lazo et al., } 2007 \\
\text { 3. Lucas et al., } 2007 \\
\text { 4. Lazo et al., } 2007 \\
\text { 5. Lucas et al., } 2007 \\
\text { 6. Diabate et al., } 2007 \\
\text { 7. Arnsten et al., } 2007 \\
\text { 8. Lazo et al., } 2007 \\
\text { 9. Nilsson-Schonnesson et al., } 2007 \\
\text { 10. Shah et al., } 2007 \\
\text { 11. Lazo et al., } 2007 \\
\text { 12. Lucas et al., } 2007 \\
\text { 13. Arnsten et al., } 2007 \\
\text { 14. Shah et al., } 2007 \\
\text { 15 Shah et al., } 2007 \\
\text { 16. Nilsson-Schonnesson et al., } 2007 \\
\text { 17. Shah et al., } 2007 \\
\text { 18. Nilsson-Schonnesson et al., } 2007 \\
\text { 19. Shah et al., } 2007 \\
\text { 20. Nilsson-Schonnesson et al., } 2007\end{array}$ & $\begin{array}{l}1.10 \\
1.18 \\
1.30 \\
1.34 \\
1.40 \\
1.43 \\
1.57 \\
1.59 \\
1.78 \\
1.81 \\
1.85 \\
1.90 \\
1.98 \\
2.09 \\
2.24 \\
2.33 \\
2.69 \\
2.71 \\
3.08 \\
3.29 \\
1.63\end{array}$ & 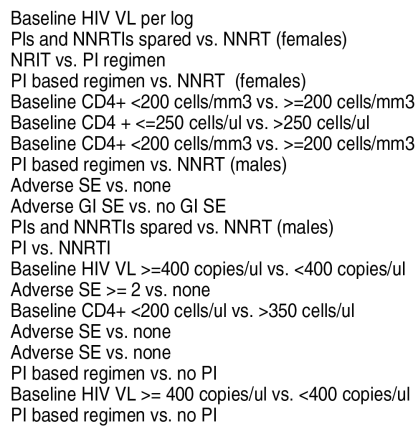 \\
\hline
\end{tabular}

\begin{tabular}{|c|c|c|c|}
\hline \multicolumn{4}{|c|}{ Statistics for each study } \\
\hline $\begin{array}{l}\text { Lower } \\
\text { limit }\end{array}$ & $\begin{array}{l}\text { Upper } \\
\text { limit }\end{array}$ & Z-Value & $\mathrm{p}$-Value \\
\hline 0.83 & 1.46 & 0.67 & 0.50 \\
\hline 0.90 & 1.55 & 1.18 & 0.24 \\
\hline 0.69 & 2.46 & 0.81 & 0.42 \\
\hline 1.11 & 1.62 & 3.03 & 0.00 \\
\hline 0.79 & 2.47 & 1.16 & 0.25 \\
\hline 1.11 & 1.83 & 2.82 & 0.00 \\
\hline 1.08 & 2.28 & 2.37 & 0.02 \\
\hline 1.12 & 2.26 & 2.57 & 0.01 \\
\hline 0.92 & 3.44 & 1.72 & 0.09 \\
\hline 1.08 & 3.04 & 2.24 & 0.02 \\
\hline 1.24 & 2.76 & 3.01 & 0.00 \\
\hline 1.16 & 3.10 & 2.57 & 0.01 \\
\hline 1.25 & 3.14 & 2.90 & 0.00 \\
\hline 1.07 & 4.09 & 2.16 & 0.03 \\
\hline 0.99 & 5.08 & 1.93 & 0.05 \\
\hline 0.76 & 7.17 & 1.47 & 0.14 \\
\hline 1.39 & 5.21 & 2.94 & 0.00 \\
\hline 0.96 & 7.64 & 1.89 & 0.06 \\
\hline 1.65 & 5.74 & 3.54 & 0.00 \\
\hline 1.72 & 6.30 & 3.59 & 0.00 \\
\hline 1.43 & 1.85 & 7.46 & 0.00 \\
\hline
\end{tabular}

0.01
Odds ratio and $95 \% \mathrm{Cl}$

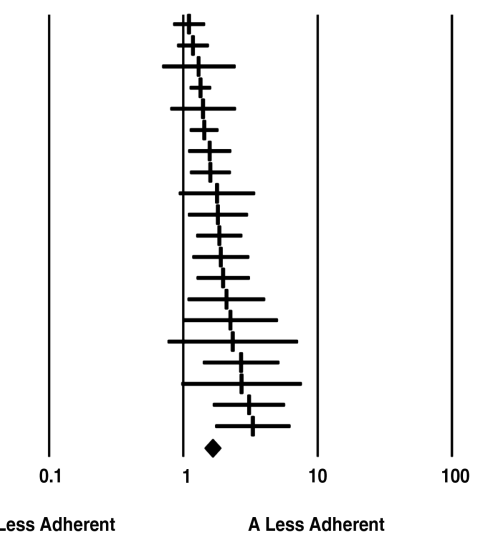

${ }^{\mathrm{a}}$ Note: The index group (A) and reference group (B) used for odds ratio (OR) computations for each statistic are listed in the Comparison column. 
Table 4. Comorbid Predictors of Medication Nonadherence ${ }^{a}$

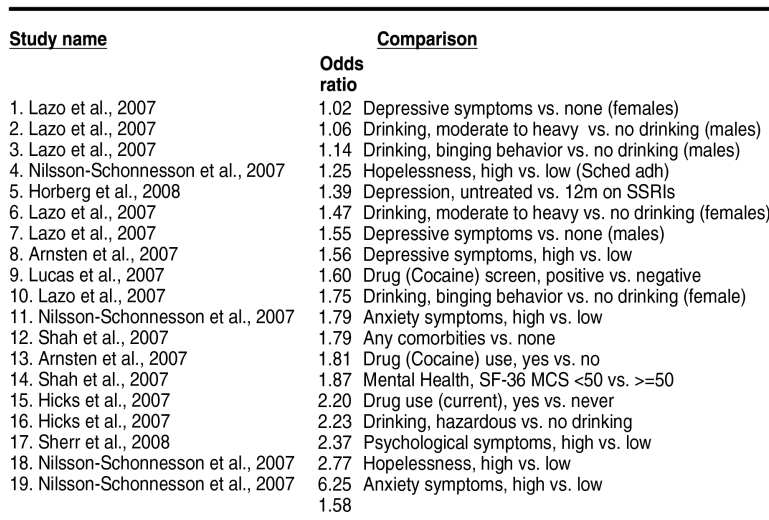

\begin{tabular}{lllll}
\multicolumn{5}{l}{ Statistics for each study } \\
$\begin{array}{c}\text { Lower } \\
\text { Limit }\end{array}$ & $\begin{array}{l}\text { Upper } \\
\text { limit }\end{array}$ & z-Value & p-Value \\
0.86 & 1.21 & 0.23 & 0.82 & \\
0.69 & 1.62 & 0.27 & 0.79 \\
0.59 & 2.20 & 0.39 & 0.70 \\
0.62 & 2.53 & 0.62 & 0.53 \\
1.18 & 1.65 & 3.90 & 0.00 \\
1.08 & 2.00 & 2.47 & 0.01 \\
1.17 & 2.05 & 3.06 & 0.00 \\
1.27 & 1.93 & 4.20 & 0.00 \\
1.03 & 2.48 & 2.10 & 0.04 \\
1.17 & 2.63 & 2.70 & 0.01 \\
0.99 & 3.23 & 1.93 & 0.05 \\
0.98 & 3.27 & 1.89 & 0.06 \\
1.12 & 2.93 & 2.42 & 0.02 \\
1.07 & 3.27 & 2.20 & 0.03 \\
1.45 & 3.34 & 3.70 & 0.00 \\
1.31 & 3.81 & 2.95 & 0.00 \\
1.26 & 4.45 & 2.68 & 0.01 \\
1.10 & 6.96 & 2.17 & 0.03 \\
2.04 & 19.16 & 3.21 & 0.00 \\
1.38 & 1.81 & 6.56 & 0.00
\end{tabular}

Odds ratio and $95 \% \mathrm{Cl}$
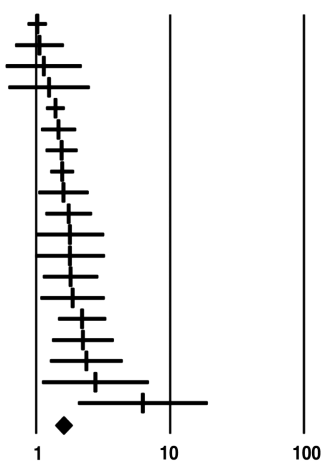

B Less Adherent

A Less Adherent

${ }^{\mathrm{a}}$ Note: The index group (A) and reference group (B) used for odds ratio (OR) computations for each statistic are listed in the Comparison column.

combined disorganizational effects of comorbities on individuals' daily lives may be synergistic, and underestimate their impact in patients with multiple mental health concerns.

Factors associated with treatment competence were consistently strong predictors of patients' medication adherence (overall OR 2.0, 95\% CI: 1.6-2.6). Within this cluster patients' optimism, understanding of HIV treatment benefits, treatment self-efficacy, and sharing treatment decision-making with their clinician were associated with increased odds that they would adhere to HIV treatment (Table 5).

The findings associated with dosing predictors show that a higher pill burden, greater versus less than 10 pills per day and to a lesser degree, a greater number of doses per day were all associated with higher nonadherence (OR: 1.5, 95\% CI: 1.31.7; Table 6).

Table 7 provides a convincing demonstration that higher levels of nonadherence are associated with higher odds of viral breakthrough. This occurred despite the use of different adherence assessment methodologies being applied across the studies. The overall OR of treatment failure across study groups was 2.0 (95\% CI: 1.6-2.5), results that are in line with the summary OR of 2.0 reported in the earlier meta-analysis of ART adherence by Simoni et al. ${ }^{2}$ and 2.3 reported by Nieuwkerk and Oort. ${ }^{3}$

Table 8 provides insight into the relative importance of some specific determinants of medication nonadherence within each of the predictive clusters. Leading the list, poor treatment competence, heavy pill burden ( $>10$ pills per day) and medication side effects are the strongest correlates of medication nonadherence. These are followed by various comorbid predictors of nonadherent behavior. Finally the number of times a day a medication is used and the number of medication classes are relatively weak correlates of medication nonadherence.

\section{Discussion}

Patients' ability to adhere to rigorous and complex administration schedule is demanding. Patients should appreciate the risks of not taking their medication regularly,

Table 5. Treatment Competence Predictors of Medication Nonadherence ${ }^{a}$

\begin{tabular}{|c|c|c|c|c|c|c|}
\hline \multirow[t]{2}{*}{ Study name } & \multirow{2}{*}{\multicolumn{2}{|c|}{ Comparison }} & \multicolumn{4}{|c|}{ Statistics for each study } \\
\hline & & & $\begin{array}{c}\text { Lower } \\
\text { limit }\end{array}$ & $\begin{array}{l}\text { Upper } \\
\text { limit }\end{array}$ & Z-Value & $\mathrm{p}-\mathrm{V}$ \\
\hline 1. Sherr et al., 2008 & 1.15 & Tx optimism, No vs. yes & 0.63 & 2.11 & 0.46 & \\
\hline 2. Diabate et al., 2007 & 1.26 & Lack of tx optimism vs. optimism & 1.01 & 1.58 & 2.02 & \\
\hline 3. Arnsten et al., 2007 & 1.66 & Education Level, HS Educ vs. < Grade 12 & 1.16 & 2.38 & 2.75 & \\
\hline 4. Beach et al., 2007 & 1.77 & Tx decision-making, doctor decides vs. shared & 1.25 & 2.50 & 3.25 & \\
\hline 5. Shah et al., 2007 & 1.82 & Satisfaction with care, low vs. high & 0.79 & 4.21 & 1.40 & \\
\hline 6. Schonnesson et al., 2007 & 2.38 & Belief in ART benefits, weak vs. strong & 1.04 & 5.45 & 2.05 & \\
\hline 7. Arnsten et al., 2007 & 2.47 & Tx self-efficacy, low vs. high & 1.87 & 3.26 & 6.41 & \\
\hline 8. Schonnesson et al., 2007 & 2.74 & Belief in HIV hlth concerns, weak vs. strong & 1.05 & 7.13 & 2.07 & \\
\hline 9. Arnsten et al., 2007 & 3.46 & Positive attitude towards ART, low vs. high & 2.09 & 5.73 & 4.82 & \\
\hline 10. Schonnesson et al., 2007 & 3.94 & Belief in ART benefits, weak vs. strong & 1.12 & 13.84 & 2.14 & \\
\hline 11. shah et al., 2007 & 4.15 & Tx Self-Efficacy, confusion vs. no confusion & 1.28 & 13.50 & 2.36 & \\
\hline & & & 1.55 & 2.57 & 5.31 & \\
\hline
\end{tabular}

${ }^{\text {a}}$ Note: The index group (A) and reference group (B) used for odds ratio (OR) computations for each statistic are listed in the Comparison column. 
Table 6. Dosing Predictors of Medication Nonadherence ${ }^{a}$

\begin{tabular}{ll}
\hline Study name & $\begin{array}{l}\text { Comparison } \\
\text { ratio }\end{array}$ \\
& 1.12 Dosing, twice vs. once daily \\
1. Boyle et al., 2008 & 1.20 Med classes, $>=4$ vs. $<4$ (males) \\
2. Lazo et al., 2007 & 1.24 Dosing, twice vs. once daily \\
3. Boyle et al., 2008 & 1.26 Med classes, $>=4$ vs. $<4$ (females) \\
4. Lazo et al., 2007 & 1.47 Pills/day, $>=10$ pills vs. $<10$ pills \\
5. Diabate et al., 2007 & 1.51 Dosing, twice vs. once daily \\
6. Boyle et al., 2008 & 1.70 Dosing, twice vs. once daily \\
7. Lucas et al., 2007 & 1.72 Pills/day, $>=3$ pills vs. $1-2$ pills \\
8. Shah et al., 2007 & 1.96 Pills/day, $7-10$ pills vs. $<=4$ doses/day \\
9. Paasche-Orlow et al., 2006 & 2.07 Med classes, $1-2$ classes vs. $<=3$ classes \\
10. Shah et al., 2007 & 2.63 Pills/day, $>10$ pills vs. $<=10$ pills \\
11. Nilson-Schonnesson et al., 2007 & 3.15 Pills/day, $>10$ pills vs. $<=10$ pills \\
12. Nilson-Schonnesson et al., 2007 & 1.48
\end{tabular}

\begin{tabular}{cccc}
\multicolumn{4}{c}{ Statistics for each study } \\
$\begin{array}{c}\text { Lower } \\
\text { limit }\end{array}$ & $\begin{array}{c}\text { Upper } \\
\text { limit }\end{array}$ & Z-Value & p-Value \\
0.76 & 1.66 & 0.56 & 0.57 \\
0.90 & 1.59 & 1.26 & 0.21 \\
0.81 & 1.89 & 1.00 & 0.32 \\
1.01 & 1.57 & 2.08 & 0.04 \\
1.14 & 1.90 & 2.93 & 0.00 \\
0.97 & 2.36 & 1.82 & 0.07 \\
1.11 & 2.61 & 2.42 & 0.02 \\
1.00 & 2.95 & 1.97 & 0.05 \\
1.08 & 3.57 & 2.20 & 0.03 \\
1.10 & 3.88 & 2.27 & 0.02 \\
1.03 & 6.72 & 2.02 & 0.04 \\
1.71 & 5.80 & 3.68 & 0.00 \\
1.28 & 1.71 & 5.34 & 0.00
\end{tabular}

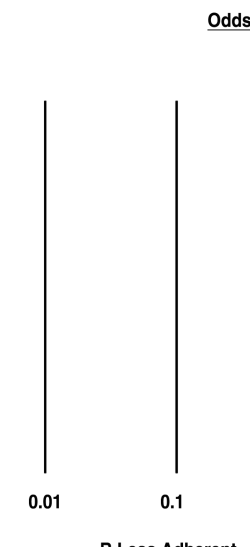

dds ratio and $95 \% \mathrm{C}$

B Less Adherent

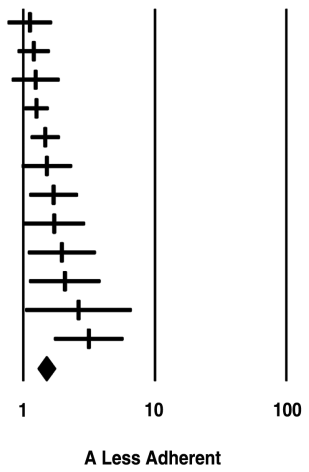

${ }^{a}$ Note: The index group (A) and the reference group (B) used for odds ratio (OR) computations for each statistic are listed in the Comparison column.

understand how and when to use each medication, and be organized enough to do this several times a day, with little room for error. Within each predictive cluster are findings that contribute to our understanding of why people occasionally, and sometime regularly, have difficulties with precise and consistent medication self-management.

\section{Cluster 1: Clinical predictors of nonadherence}

The clinical predictors of nonadherence suggest that persons with high baseline viral loads and compromised immune function at baseline were more likely to be nonadherent to their future HIV treatment regimen. It is possible that the effects of earlier nonadherence resulted in poorer outcomes and this behavior was simply carried forward with subsequent treatment. As expected, the number and type of side effects, and particularly those associated with PI-based treatments, exert a consistent negative effect on adherence behavior. Nonadherence estimates comparing naïve versus experienced ART patients appear to vary greatly; possibly because of the amount of education naïve patients receive when initiating HIV therapy for the first time. Supporting this idea, changes in adherence levels have been shown over time among ARTnaïve patients but not among experienced patients. ${ }^{27}$

\section{Cluster 2: Comorbid predictors of nonadherence}

The comorbidities of alcohol/drug abuse, depression and anxiety consistently predicted nonadherence behavior. Given the high degree of organization and discipline required to

Table 7. Nonadherence Levels Predicting Viral Breakthrough ${ }^{\mathrm{a}}$

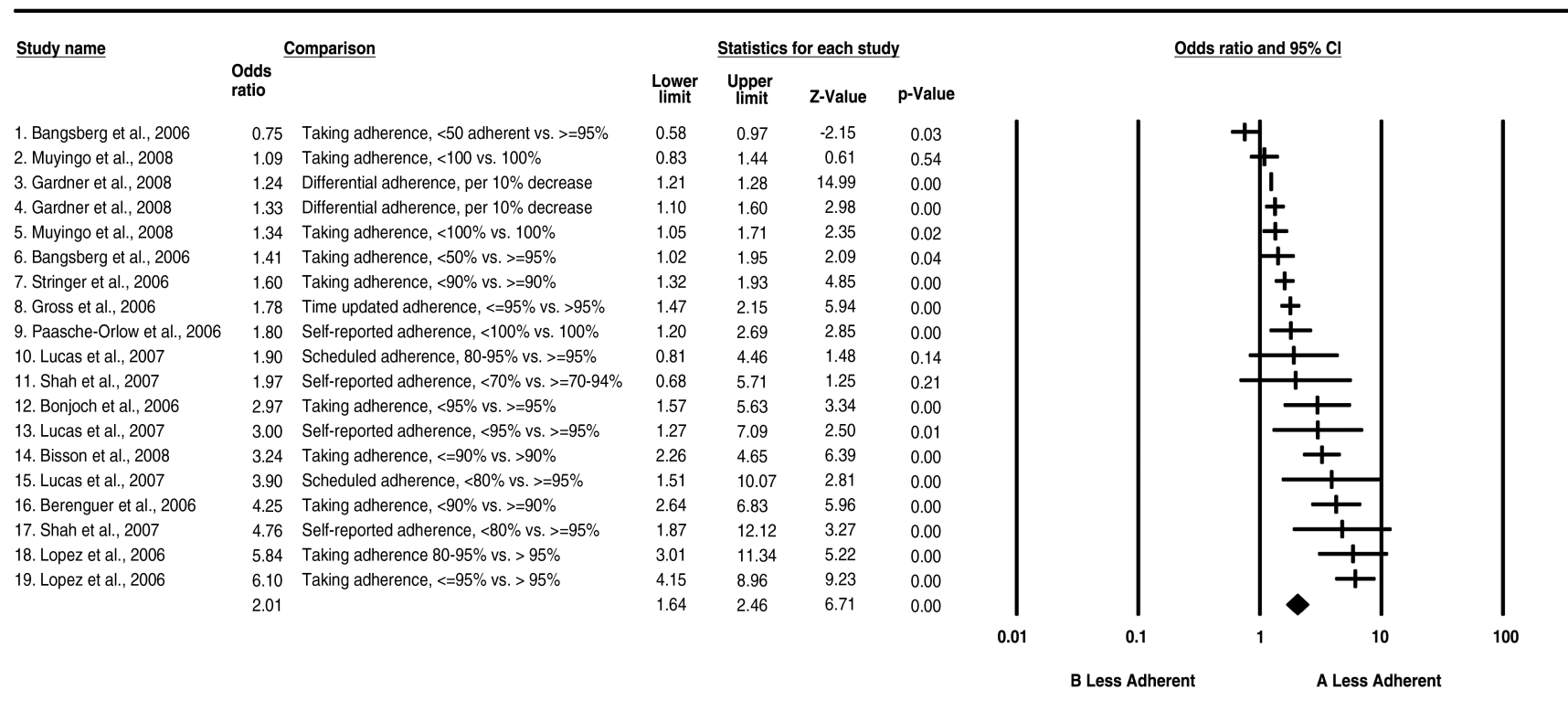

${ }^{a}$ Note: The index group (A) and reference group (B) used for odds ratio (OR) computations for each statistic are listed in the Comparison column. 
Table 8. Rank Ordered Effects of Specific Determinants of Treatment Nonadherence

\begin{tabular}{lrr}
\hline Predictor of nonadherence & Pooled OR (95\% CI) & Reference studies \\
\hline Poor medication self-management, low Tx self-efficacy & $2.4(2.0-3.0)$ & $(24,28,42,53)$ \\
High pill burden (more vs. less than 10 pills daily) & $2.0(1.4-3.0)$ & $(40,47,52)$ \\
Frequent or severe medication side effects & $1.9(1.4-2.7)$ & $(24,40)$ \\
Anxiety or depression & $1.6(1.3-1.9)$ & $(24,27,28,40,50)$ \\
Alcohol or substance abuse & $1.6(1.4-1.8)$ & $(28,49,50,51)$ \\
Twice vs. once daily dosing & $1.4(1.0-1.7)$ & $(46,51)$ \\
Use of more medication classes $(>3$ vs. $\leq 3)$ & $1.3(1.1-1.6)$ & $(24,50)$ \\
\hline
\end{tabular}

OR, odds ratio; $\mathrm{CI}$, confidence interval; $\mathrm{Tx}$, treatment.

remain highly compliant, it is possible that the underlying causes of nonadherence among binge drinkers or drug users are impulsivity, avoidance and inadequate planning. This may not be the same for persons who are depressed and/or anxious, for whom feelings of anxiety about coping with life and hopeless attributions may affect motivation associated with effective medication management, ultimately with undesirable clinical outcomes.

\section{Cluster 3: Treatment competence predictors of nonadherence}

Patients' lack of ART competence appeared as the strongest nonadherence predictors; with poorer medication selfefficacy, incorrect beliefs about the treatment and the disease, lower optimism, and poorer understanding of treatment were associated with worse medication adherence. This cluster speaks to the central importance of patients being informed, actively involved in the treatment process, and competent to manage their own treatment. Moreover, such competencies are clearly essential to good clinical outcomes.

\section{Cluster 4: Dosing predictors of nonadherence}

This set of predictors was ranked as second in importance to treatment competence. The complex administration requirements associated with many medication regimens (e.g., a large number of pills, different types of medications with differing dietary requirements) often result in doses being delayed, missed, or not taken with the right food. The value of efforts to simplify dosing is demonstrated in studies showing that providing a pillbox organizer almost doubles the odds of patients remaining adherent while on complex regimens. ${ }^{55}$ The relatively minor difference between once versus twice daily dosing seemed to be a weak predictor of medication nonadherence.

\section{Cluster 5: Nonadherence as a predictor of treatment failure}

This cluster underscores the general clinical importance of medication adherence in the effective treatment of HIV/AIDS. Interestingly, in contrast to predictors of nonadherence, the results presented in Table 7 came from a set of more technical and clinically focused research studies. These results provide evidence of the dose-response relationship between level of nonadherence and the odds of treatment failure. Notably, the summary OR for this cluster would have been still higher if the per unit nonadherence OR estimates, such as those reported by Gardner et al. ${ }^{39}$ were omitted.

\section{Summary}

It was encouraging that some of the recent adherence research had acted on the earlier recommendations by Simoni et al., ${ }^{2}$ providing results that discriminate between the various forms of nonadherence. Such discrimination allows for a deeper understanding of the causes of nonadherence and their relative importance among different patient subgroups. The current findings may help inform the design of educational interventions to address specific determinants of nonadherence that lead to undesirable clinical outcomes. Moreover, the use of differential nonadherence estimates (i.e., for each medication class) could be used to identify the relative importance of specific aspects of nonadherence as they impact medication failure, thereby informing directions for drug development. ${ }^{56}$

The strength of relationships between predictors of nonadherence and observed nonadherence are often subject to great variation based on the chosen referent group used to compute the OR estimates. It is difficult to infer magnitude of effects associated with medication nonadherence from any particular study from the effects of researchers' choice of patient reference group, medication type/regimen, and method of adherence assessment. Across a larger number of studies, these methodological effects are averaged out.

This analysis did not apply meta-analytic regression or analysis of variance (ANOVA) methods to model the effects of estimate variation across studies. Such an approach might prove informative, although despite efforts to standardize, some problems might persist due to constraints inherent in the individual study design. For example, longer retrospective cohort studies often confound length of time on a particular therapy with nonadherence.

There is some room for improvement within the field with respect to consistent reporting of data and effect size estimates. In situations where a linear trend or dose-response is observed between the causes of nonadherence and nonadherence it would be helpful to describe the trend per unit change in the predictor, as is frequently reported regarding viral load and some dimensions of comorbidity. For example, some authors have taken steps to standardize the doseresponse of nonadherence level on clinical outcomes by reporting ORs per $10 \%$ decline in observed adherence.

\section{Limitations of the current review and future research}

This review was not intended to be a comprehensive summary of all articles in the field. It was intended to clearly represent the effects of various clinical, medication-related, 
and patient factors on nonadherence that have been reported within recent HIV treatment literature. The relatively small number of studies on which some of the comparisons are based may not be sufficient to provide precise effect size estimates, and more study may be required.

\section{Acknowledgments}

Funding for this empirical review was funded through an independent grant from Merck \& Co. Inc.

\section{Author Disclosure Statement}

No competing financial interests exist.

\section{References}

1. Moore RD, Keruly JC, Gebo KA, Lucas GM. An improvement in virologic response to highly active antiretroviral therapy in clinical practice from 1996 through 2002. J Acquir Immune Defic Syndr 2005;39:195-198.

2. Simoni JM, Kurth AE, Pearson CR, Pantalone DW, Merrill JO, Frick PA. Self-report measures of antiretroviral therapy adherence: A review with recommendations for HIV research and clinical management. AIDS Behav 2006;10:227245.

3. Nieuwkerk PT, Oort FJ. Self-reported adherence to antiretroviral therapy for HIV-1 infection and virologic treatment response: A meta-analysis. J Acquir Immune Defic Syndr 2005;38:445-448.

4. Le Moing V, Rabaud C, Journot V, et al. Incidence and risk factors of bacterial pneumonia requiring hospitalization in HIV-infected patients started on a protease inhibitorcontaining regimen. HIV Med 2006;7:261-267.

5. Castro KG, Ward JW, Slutsker L, Buehler JW, Jaffe HW, Berkelman RL. Revised Classification System for HIV Infection and Expanded Surveillance Case Definition for AIDS Among Adolescents and Adults. National Center for Infectious Diseases Division of HIV/AIDS, 1993. www .cdc.gov $/ \mathrm{mmwr} /$ preview $/ \mathrm{mmwrhtml} / 00018871$.htm (Last accessed June 11, 2009).

6. Bangsberg DR, Acosta EP, Gupta R, et al. Adherenceresistance relationships for protease and non-nucleoside reverse transcriptase inhibitors explained by virological fitness. AIDS 2006;20:223-231.

7. Tozzi V, Zaccarelli M, Bonfigli S, et al. Drug-class-wide resistance to antiretrovirals in HIV-infected patients failing therapy: Prevalence, risk factors and virological outcome. Antivir Ther 2006;11:553-560.

8. Proctor VE, Tesfa A, Tompkins DC. Barriers to adherence to highly active antiretroviral therapy as expressed by people living with HIV/AIDS. AIDS Patient Care STDs 1999;13: 535-544.

9. Bangsberg DR, Hecht FM, Charlebois ED, et al. Adherence to protease inhibitors, HIV-1 viral load, and development of drug resistance in an indigent population. AIDS 2000;14:357366.

10. Haubrich RH, Little SJ, Currier JS, et al. The value of patientreported adherence to antiretroviral therapy in predicting virologic and immunologic response. California Collaborative Treatment Group. AIDS 1999;13:1099-1107.

11. Nieuwkerk PT, Sprangers MA, Burger DM, et al. Limited patient adherence to highly active antiretroviral therapy for HIV-1 infection in an observational cohort study. Arch Intern Med 2001;161:1962-1968.
12. Friedland GH, Williams A. Attaining higher goals in HIV treatment: The central importance of adherence. AIDS 1999;13(Suppl 1):S61-72.

13. Barreiro P, Garcia-Benayas T, Soriano V, Gallant J. Simplification of antiretroviral treatment-How to sustain success, reduce toxicity and ensure adherence avoiding PI use. AIDS Rev 2002;4:233-241.

14. Nachega JB, Hislop M, Dowdy DW, Chaisson RE, Regensberg L, Maartens G. Adherence to nonnucleoside reverse transcriptase inhibitor-based HIV therapy and virologic outcomes. Ann Intern Med 2007;146:564-573.

15. Molina JM, Podsadecki TJ, Johnson MA, et al. A lopinavir/ritonavir-based once-daily regimen results in better compliance and is non-inferior to a twice-daily regimen through 96 weeks. AIDS Res Hum Retroviruses 2007;23:1505-1514.

16. Nachega JB, Hislop M, Dowdy DW, et al. Adherence to highly active antiretroviral therapy assessed by pharmacy claims predicts survival in HIV-infected South African adults. J Acquir Immune Defic Syndr 2006;43:78-84.

17. Cooper CL, van Heeswijk RP. Once-daily nevirapine dosing: A pharmacokinetics, efficacy and safety review. HIV Med 2007;8:1-7.

18. Vrouenraets SM, Wit FW, van Tongeren J, Lange JM. Efavirenz: A review. Expert Opin Pharmacother 2007;8:851-871.

19. Malta M, Magnanini MM, Strathdee SA, Bastos FI. Adherence to antiretroviral therapy among HIV-infected drug users: A meta-analysis. AIDS Behav (ahead of print). DOI: 10.1007/S1046-0089489-7.

20. Malta M, Strathdee SA, Magnanini MM, Bastos FI. Adherence to antiretroviral therapy for human immunodeficiency virus/ acquired immune deficiency syndrome among drug users: A systematic review. Addiction 2008;103:1242-1257.

21. Herrmann S, McKinnon E, John M, et al. Evidence-based, multifactorial approach to addressing non-adherence to antiretroviral therapy and improving standards of care. Intern Med J 2008;38:8-15.

22. Mills EJ, Nachega JB, Buchan I, et al. Adherence to antiretroviral therapy in sub-Saharan Africa and North America: A meta-analysis. JAMA 2006;296:679-690.

23. Muyingo SK, Walker AS, Reid A, et al. Patterns of individual and population-level adherence to antiretroviral therapy and risk factors for poor adherence in the first year of the DART trial in Uganda and Zimbabwe. J Acquir Immune Defic Syndr 2008;48:468-475.

24. Shah B, Walshe L, Saple DG, et al. Adherence to antiretroviral therapy and virologic suppression among HIVinfected persons receiving care in private clinics in Mumbai, India. Clin Infect Dis 2007;44:1235-1244.

25. Wanchu A, Kaur R, Bambery P, Singh S. Adherence to generic reverse transcriptase inhibitor-based antiretroviral medication at a tertiary center in North India. AIDS Behav 2007;11:99-102.

26. Dilorio C, McDonnell M, McCarty F, Yeager K. Initial testing of the Antiretroviral Medication Complexity Index. J Assoc Nurses AIDS Care 2006;17:26-36.

27. Horberg MA, Silverberg MJ, Hurley LB, et al. Effects of depression and selective serotonin reuptake inhibitor use on adherence to highly active antiretroviral therapy and on clinical outcomes in HIV-infected patients. J Acquir Immune Defic Syndr 2008;47:384-390.

28. Arnsten JH, Li X, Mizuno Y, et al. Factors associated with antiretroviral therapy adherence and medication errors among HIV-infected injection drug users. J Acquir Immune Defic Syndr 2007;46(Suppl 2):S64-71. 
29. Reynolds NR, Sun J, Nagaraja HN, Gifford AL, Wu AW, Chesney MA. Optimizing measurement of self-reported adherence with the ACTG Adherence Questionnaire: A crossprotocol analysis. J Acquir Immune Defic Syndr 2007;46:402409.

30. Wise J, Operario D. Use of electronic reminder devices to improve adherence to antiretroviral therapy: A systematic review. AIDS Patient Care STDs 2008;22:495-504.

31. Grymonpre RE, Didur CD, Montgomery PR, Sitar DS. Pill count, self-report, and pharmacy claims data to measure medication adherence in the elderly. Ann Pharmacother 1998;32:749-754.

32. Arnsten JH, Demas PA, Farzadegan H, et al. Antiretroviral therapy adherence and viral suppression in HIV-infected drug users: Comparison of self-report and electronic monitoring. Clin Infect Dis 2001;33:1417-1423.

33. Wagner G, Miller LG. Is the influence of social desirability on patients' self-reported adherence overrated? J Acquir Immune Defic Syndr 2004;35:203-204.

34. Sankar AP, Nevedal DC, Neufeld S, Luborsky MR. What is a missed dose? Implications for construct validity and patient adherence. AIDS Care 2007;19:775-780.

35. Pearson CR, Simoni JM, Hoff P, Kurth AE, Martin DP. Assessing antiretroviral adherence via electronic drug monitoring and self-report: An examination of key methodological issues. AIDS Behav 2007;11:161-173.

36. Deschamps AE, De Geest S, Vandamme AM, Bobbaers H, Peetermans WE, Van Wijngaerden E. Diagnostic value of different adherence measures using electronic monitoring and virologic failure as reference standards. AIDS Patient Care STDs 2008;22:735-743.

37. Liu H, Golin CE, Miller LG, et al. A comparison study of multiple measures of adherence to HIV protease inhibitors. Ann Intern Med 2001;134:968-977.

38. Sherr L, Lampe F, Norwood S, et al. Adherence to antiretroviral treatment in patients with HIV in the UK: A study of complexity. AIDS Care 2008;20:442-448.

39. Gardner EM, Sharma S, Peng G, et al. Differential adherence to combination antiretroviral therapy is associated with virological failure with resistance. AIDS 2008;22:75-82.

40. Nilsson Schonnesson L, Williams ML, Ross MW, Bratt G, Keel B. Factors associated with suboptimal antiretroviral therapy adherence to dose, schedule, and dietary instructions. AIDS Behav 2007;11:175-183.

41. Lopez JC, Moreno S, Jimenez-Onate F, Clotet B, Rubio R, Hernandez-Quero J. A cohort study of the food effect on virological failure and treatment discontinuation in patients on HAART containing didanosine enteric-coated capsules (FOODDIe Study). HIV Clin Trials 2006;7:155-162.

42. Beach MC, Duggan PS, Moore RD. Is patients' preferred involvement in health decisions related to outcomes for patients with HIV? J Gen Intern Med 2007;22:1119-1124.

43. Berenguer J, Perez-Elias MJ, Bellon JM, et al. Effectiveness and safety of abacavir, lamivudine, and zidovudine in antiretroviral therapy-naive HIV-infected patients: results from a large multicenter observational cohort. J Acquir Immune Defic Syndr 2006;41:154-159.

44. Bisson GP, Gross R, Bellamy S, et al. Pharmacy refill adherence compared with CD4 count changes for monitoring
HIV-infected adults on antiretroviral therapy. PLoS Med 2008;5:e109.

45. Bonjoch A, Paredes R, Domingo P, et al. Long-term safety and efficacy of nevirapine-based approaches in HIV type 1infected patients. AIDS Res Hum Retroviruses 2006;22:321329.

46. Boyle BA, Jayaweera D, Witt MD, Grimm K, Maa JF, Seekins DW. Randomization to once-daily stavudine extended release/ lamivudine/efavirenz versus a more frequent regimen improves adherence while maintaining viral suppression. HIV Clin Trials 2008;9:164-176.

47. Diabate S, Alary M, Koffi CK. Determinants of adherence to highly active antiretroviral therapy among HIV-1-infected patients in Cote d'Ivoire. AIDS 2007;21:1799-1803.

48. Gross R, Yip B, Lo Re V 3rd, et al. A simple, dynamic measure of antiretroviral therapy adherence predicts failure to maintain HIV-1 suppression. J Infect Dis 2006;194:1108-1114.

49. Hicks PL, Mulvey KP, Chander G, et al. The impact of illicit drug use and substance abuse treatment on adherence to HAART. AIDS Care 2007;19:1134-1140.

50. Lazo M, Gange SJ, Wilson TE, et al. Patterns and predictors of changes in adherence to highly active antiretroviral therapy: Longitudinal study of men and women. Clin Infect Dis 2007;45:1377-1385.

51. Lucas GM, Mullen BA, McCaul ME, Weidle PJ, Hader S, Moore RD. Adherence, drug use, and treatment failure in a methadone-clinic-based program of directly administered antiretroviral therapy. AIDS Patient Care STDs 2007;21:564-574.

52. Paasche-Orlow MK, Cheng DM, Palepu A, Meli S, Faber V, Samet JH. Health literacy, antiretroviral adherence, and HIV-RNA suppression: A longitudinal perspective. J Gen Intern Med 2006;21:835-840.

53. Schonnesson LN, Williams ML, Ross MW, Diamond PM, Keel B. Three types of adherence to HIV antiretroviral therapy and their association with AIDS diagnosis, medication side-effects, beliefs about antiretroviral therapy, and beliefs about HIV disease. Int J STD AIDS 2007;18:369373.

54. Stringer JS, Zulu I, Levy J, et al. Rapid scale-up of antiretroviral therapy at primary care sites in Zambia: Feasibility and early outcomes. JAMA 2006;296:782-793.

55. Petersen ML, Wang Y, van der Laan MJ, Guzman D, Riley E, Bangsberg DR. Pillbox organizers are associated with improved adherence to HIV antiretroviral therapy and viral suppression: A marginal structural model analysis. Clin Infect Dis 2007;45:908-915.

56. Sun J, Nagaraj HN, Reynolds NR. Discrete stochastic models for compliance analysis based on an AIDS Clinical Trial Group (ACTG) study. Biom J 2007;49:731-741.

Address correspondence to: Mark J. Atkinson, M.Ed., Ph.D. Health Services Research Center University of California at San Diego 5440 Morehouse Drive, Suite 3500 San Diego, CA 92121

E-mail: mjatkinson@ucsd.edu 